\title{
DEVELOPMENT OF FORMS OF STRUCTURAL MATHEMATICAL MODELING IN DYNAMICS OF MECHANICAL OSCILLATION SYSTEMS: IMPEDANCE AND POSSIBLE VARIANTS OF APPROACH
}

\author{
S.V. Eliseev ${ }^{I}$, V.B. Kashuba ${ }^{2}$, S.K. Kargapoltsev ${ }^{l}$ and A.V. Nikolaev ${ }^{I}$
}

1 Irkutsk State Transport University, Irkutsk, Russia Federation 2 Bratsk State University, Bratsk, Russia Federation e-mail:eavsh@ya.ru

\begin{abstract}
Offering method of transformation of mathematical models of oscillation systems based on using of structural schemes. Structural analogs of mathematical models used for creature of variants of transfer functions of initial mechanical systems. Technology of transformation of structural schemes with object which has properties of integrated link of second order. Is shown that impedance is local characteristic of frequence dynamical properties of mechanical oscillation systems, in particular, vibration protection systems. Characteristics-analogs of impedance are given. They constructed on base using of movements and accelerations of allocated points. Conditions of ties of relative frequence characteristics are given.

Conditions of compatibility of structural models of mechanical chains theory and automation control theory are identified.
\end{abstract}

Keyboards: impedance, structural scheme, structural mathematical model, transfer function, structural transformations;

\section{INTRODUCTION}

Design scheme in view of mechanical chain and structural schemes of equivalent in dynamical ratio of automation control systems are widely used in solving tasks of machines mechanics $[4,9,10,16]$. Interesting applications of structural theory appeared in solving of tasks of vibration protection of machines and equipment, transport dynamics that reflected in a number of papers $[2,13,17]$.

Representation of technical objects in view of structural mathematical models has range of advantages over usual approaches because of simplicity of ideas of arising of dynamical ties. Such ties arise at connecting between themselves of typical elementary links of oscillation systems. In general, develop of structural mathematical modeling allowed to enter in considering of range of new ideas about expansion of set of typical elements of vibroprotection systems, quasi-springs, lever ties and etc, [3,6,14]. Less developed approaches base on using of technology of application of impedances but structural models has some potential in in this plane 
$[1,7,18]$.

Generalized approach to choice of transfer forms is developed in offering article. Such transfer forms are obtained of structural schemes with possibilities of display of system dynamical properties.

\section{General positions. Formulation of the tasks of research.}

Design scheme of vibroprotection system in view of chain mechanical oscillation system with two freedom degrees is presented on fig. 1 a $\div$ e. Rigid body $m_{1}$ or $m_{2}$ depending from features of tasks of dynamical synthesis can act in quality of protection object. System on fig. 1a has linear elastic elements $k_{1}, k_{2}, k_{3}$. Friction forces is assumed to be small. External power $Q_{1}, Q_{2}$ and kinematical $z_{1}, z_{2}$ influence are harmonically. Structural scheme or structural mathematical model is given on fig. 1b. Such model has detailed system of straight and feedback ties and goes into coordinate system $\bar{y}_{1}, \bar{y}_{2}$ in fixed basis. All necessary intermediate stages of creature of mathematical models in view of differential equations of movement, necessary transformation of Laplace and creatures of structural schemes is produced in accordance with $[4,10,16]$. Icon $(-)$ on variables means depiction on Laplace; $p=j \omega$ is complex variable $(j=\sqrt{ }-1)$.

Structural scheme on fig. $1 \mathrm{~b}$ can be converted to more compact view (fig. 1c) where partial systems and ties bet ween them are allocated. In this case ties between partial systems are called as elastic. Mathematical model in view of system of two differential linear equations is shown on fig. $1 \mathrm{~d}$. Structural schemes on fig. 1b and fig. 1c are graphic analogs of mathematical model on fig. 1d. Structural scheme on symbolic depiction of mechanical chains theory is represented on fig. 1e [4]. Two types of external disturbances are shown on fig. 1e.

Analysis of dynamical possibilities of oscillation systems connects with identification of coerced stiffnesses. Such stiffness's correlated with certain branches of mechanical system. Possibilities of analytical approaches in estimation of dynamical properties was considered in articles $[4,9,10,16]$. Dynamical properties are based on using of transfer function when entrance influence is external disturbance (force or kinematical) and exit disturbance is coordinate of displacement of object. Impedances are used in range of tasks which connects with estimation of dynamical properties. Impedances theory bases on electro-mechanical analogies (current velocity, voltage - force) $[4,5]$.

In offering research generalized approach is developed. Within the such approach impedance is considered as some local (or individual characteristics) which use in realizations of methods of structural mathematical modeling. 


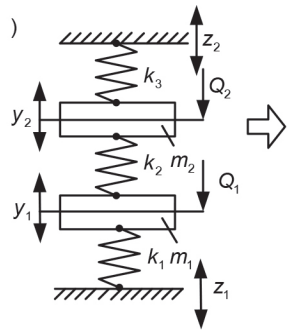

c)

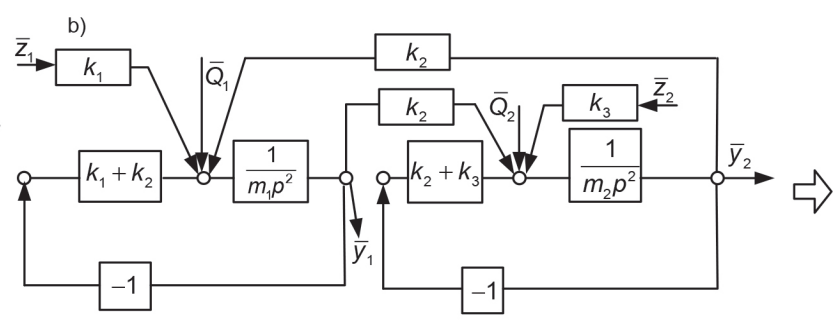

d)
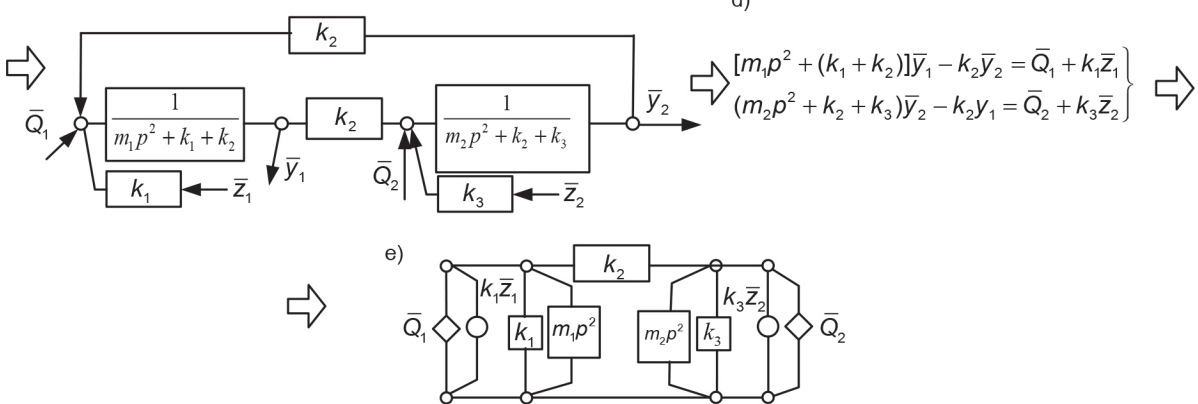

Fig. 1. Design and structural schemes of vibroprotection systems with two freedom degrees: a) design scheme; b) structural detailed scheme; c) structural scheme with allocation of partial systems; d) mathematical model of system on fig. 1a; e) structural scheme of mechanical chain which is equivalent structural scheme on fig. $1 \mathrm{~b}$

\section{Estimation of dynamical possibilities of vibroprotection systems with using of structural mathematical models.}

For simplification we will consider a number of private positions with a choice of parameters of the design scheme on fig. 1a.
1. If $\overline{\mathrm{Q}}_{2}=0, \overline{\mathrm{Z}}_{1}=0, \overline{\mathrm{Z}}_{2}=0$, but $\overline{\mathrm{Q}}_{1} \neq 0$ , then coerced stiffness of generalized spring (object $m_{1}$ rests on such spring) determined of equation

$$
K_{c 1}=\left(\frac{\left(m_{2} p^{2}+k_{3}\right) k_{2}}{m_{2} p^{2}+k_{2}+k_{3}}\right)+k_{1}=\frac{m_{2} p^{2}\left(k_{1}+k_{2}\right)+k_{1} k_{2}+k_{1} k_{3}+k_{2} k_{3}}{m_{2} p^{2}+k_{2}+k_{3}}
$$

that follows from mechanical chain on fir. 1e.

2. Coerced stiffness of generalized spring from scheme on fig. 1c can be found in view

$$
\begin{gathered}
\mathrm{K}_{\mathrm{c} 1}=\mathrm{k}_{1}-\frac{\mathrm{k}_{2}^{2}}{\left(\mathrm{~m}_{2} \mathrm{p}^{2}+\mathrm{k}_{2}+\mathrm{k}_{3}\right)}+\mathrm{k}_{2}=\frac{\mathrm{m}_{2} \mathrm{p}^{2}\left(\mathrm{k}_{1}+\mathrm{k}_{2}\right)+\mathrm{k}_{1} \mathrm{k}_{2}+\mathrm{k}_{1} \mathrm{k}_{3}+\mathrm{k}_{2} \mathrm{k}_{3}}{\mathrm{~m}_{2} \mathrm{p}^{2}+\mathrm{k}_{2}+\mathrm{k}_{3}}= \\
=\mathrm{k}_{1}+\frac{\left(\mathrm{m}_{2} \mathrm{p}^{2}+\mathrm{k}_{3}\right) \mathrm{k}_{2}}{\mathrm{~m}_{2} \mathrm{p}^{2}+\mathrm{k}_{2}+\mathrm{k}_{3}}
\end{gathered}
$$

Equations (1) and (2) match that confirms adequacy of different forms of structural models. 
3. From mathematical model (fig. 1d) follows that

$$
\bar{y}_{2}=\frac{\bar{y}_{1} k_{2}}{m_{2} p^{2}+k_{2}+k_{3}},
$$

After substitution (3) in the equation $\left(\mathrm{m}_{2} \mathrm{p}^{2}+\mathrm{k}_{1}+\mathrm{k}_{2}\right)-\mathrm{k}_{2} \overline{\mathrm{y}}_{2}=\overline{\mathrm{Q}}_{1}$, let's receive the movement equation in shape

$$
\bar{y}_{1}\left[m_{1} p^{2}+k_{1}+\frac{\left(m_{2} p^{2}+k_{3}\right) k_{2}}{m_{2} p^{2}+k_{2}+k_{3}}\right]=\bar{Q}_{1}
$$

Thus the scheme considered above conclusiveness - item 1 positions, item 2 , item 3 give the grounds for the statement about adequacy of mathematical models on positions fig. 1 (fig. 1c, fig. 1e).

The design scheme fig. 1a reflects physical features of mechanical oscillatory system in a position.

Resulted above equation are presented in the operational form received on the basis of transformations by Laplace $[4,9,10,16]$.

Let's notice that the initial design scheme (fig. 1a) can be displayed in structuresanalogues of the theory of automatic control, and also with use of analogies to electric schemes [4,5].

The first approach is developed enough and constructed on use of the dynamic analogies connected with the concept of feedback. A number of works $[10,16]$ in which the

\section{Features of identification of impedance.}

If to consider mechanical oscillatory system with one degree of freedom, as is shown in fig. 2, the relation

$$
S(p)=\frac{\bar{Q}}{\bar{y}}=j \omega m+r+\frac{k}{j \omega}
$$

is called as an impedance [7].

In work [4] impedance is called also as full complex resistance of system (CRS). We will notice that an impedance, within the limits of the structural theory vibroprotection systems transfer function of system (fig. 2), defined of the structural schemes resulted on fig. 3 a-e will be.

The structural scheme on fig. 3a has a direct mechanical oscillatory system is considered as system of automatic control of a special kind (or appointments) is known. The analytical device of the theory of automatic control is used for the decision of problems of dynamic synthesis. At the same time rules of transformation of structural schemes equivalent, in the dynamic relation of systems of automatic control, have the features. The theoretical basis in problems of dynamics of mechanical oscillatory systems extends on linear systems with corresponding possibilities of frequency methods of the analysis and synthesis [2-4,9,10,13,17].

The second approach is based on other basis which is connected with analogies between elements of electric and mechanical oscillatory systems. A number of works $[1,4,5,9,7,18]$ in which the general and private questions of synthesis of the mechanical chains which are analogues of electric chains are considered is known. In the theory of chains questions of electromechanical analogies were considered in works $[1,4,5,7,18]$. At the same time many questions yet have not received due detailed elaboration of representations about possible borders of analogies. In particular, such problems arise at research of mechanical oscillatory systems with lever communications and mechanisms. Interest to such works has found reflection in works $[3,6,8,12,15]$.

chain in which there is an object of protection $\frac{1}{\mathrm{mp}^{2}}$, and also is elastic-dissipative the block $k+b p$, representing a spring parallel connection $k$ and damper $b p$ (where $b p$ - factor of a viscous friction).

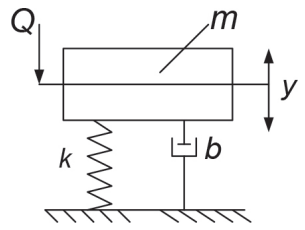

Fig. 2. Design scheme of VPD with one freedom degree 

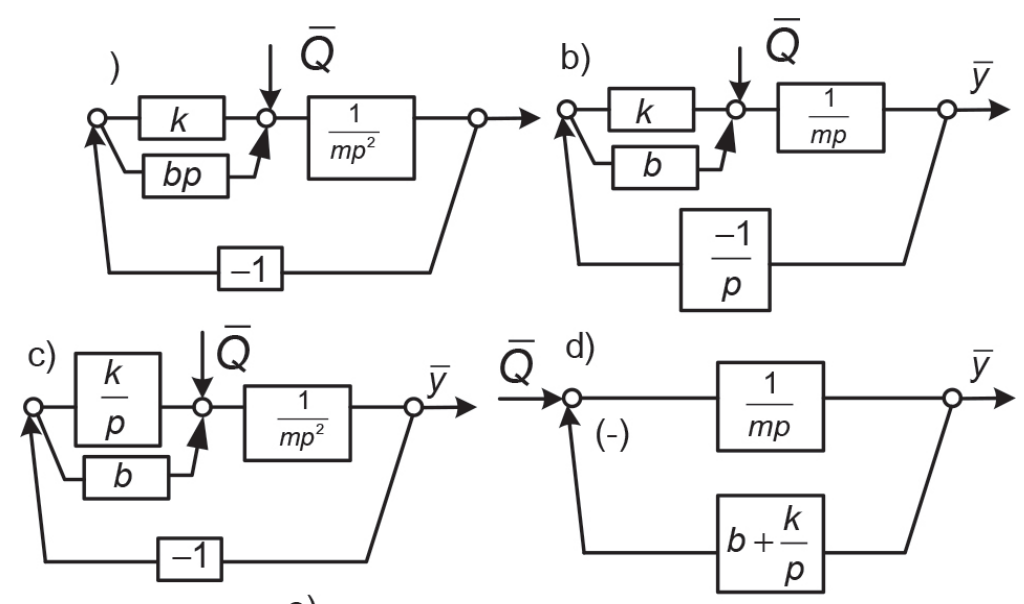

e)

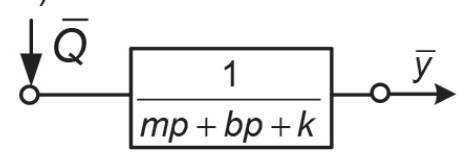

Fig. 3. Structural scheme (a) and forms of transformation of structural model for receipt of impedance:

b) - introduction of block in feedback tie; c) - transfer of link 1/p in straight chain;

d) - formation of feedback negative tie on ratio to link $1 / \mathrm{mp}^{2} ;$ e) - generalized structural model

We use the structural scheme on fig. 3e, whence transfer function at an input in the form of external power influence $\bar{Q}$ has a

$$
W(p)=\frac{\bar{v}}{\bar{Q}}=\frac{1}{m p+b+\frac{k}{p}} .
$$

Here $p=j \omega$ - the complex variable used in frequency methods of the analysis of the theory of automatic control [3], and also in target signal in the form of speed of object in weight $\mathrm{m}$, we will receive

$$
S(p)=\frac{1}{W(p)}=\frac{\bar{Q}}{\bar{y}}=m p+b+\frac{k}{p}=j \omega m+b+\frac{k}{j \omega} .
$$

Concept introduction about an impedance is connected with use of such parameter of a dynamic condition, as speed of a mass inertial element $(m)$. In physical sense the impedance characterizes dynamic properties of system, that is the response of an element in weight $\mathrm{m}$ in the form of speed $v$ on power influence.

If for definition of impedance $S(p)$ to use the structural scheme on fig. 3a then it is possible to find transfer function of a kind

$$
W(p)=\frac{\bar{y}}{\bar{Q}}=\frac{1}{m p^{2}+b p+k} .
$$

For transition to transfer function with parameter $\bar{v}=p \bar{y}[18]$, equation (8) is transformed to view 


$$
W_{2}(p)=\frac{\bar{y}}{\bar{Q}}=\frac{1}{p\left(m p+b p+\frac{k}{p}\right)},
$$

Whence follows that

$$
W_{3}(p)=\frac{p \bar{y}}{\bar{Q}}=\frac{1}{m p+b p+\frac{k}{p}} .
$$

If to make inversion (10) we will receive equation for an impedance

$$
S(p)=\frac{1}{W_{3}(p)}=m p+b+\frac{k}{p}
$$

Communication of equations (6) - between considered characteristics of dynamic (11) is predetermined by a generality of communications with the initial structural scheme (or structural mathematical model), presented on fig. 3a. structural scheme transformations open not only communications properties, but also important for structural modelling of possibility of introduction in consideration of typical elementary links VPS with transfer functions of integrating links of the first sort.

\section{About ties of impedance with other types of characteristics.}

In the structural theory of mechanical vibroprotection systems from transfer function oscillatory systems, is more exact in its appendices whereas from the structural theory

(8) follows

$$
k_{c}(p)=\frac{\bar{Q}}{\bar{y}}=\frac{1}{W_{1}(p)}=m p^{2}+b p+k,
$$

that on the physical sense corresponds to the coerced stiffness of the generalized spring. From (12) we will receive

$$
k_{c}(p)=p\left(m p^{2}+b p+\frac{k}{p}\right)=p \cdot S(p) .
$$

That is the impedance and the coerced stiffness of the generalized spring are connected among themselves by a parity

$$
k_{c}(p)=p \cdot S(p)
$$

or

$$
S(p)=\frac{k_{c}(p)}{p}
$$

In turn $S(p) \cdot \bar{v}=\vec{Q}$, a $k_{c} \cdot \bar{y}=\vec{Q}$, whence it is possible to receive that $\bar{v}^{2} \cdot S(p)=\vec{Q} \cdot \bar{v}$, a $k_{c} \cdot \bar{v} \cdot \bar{y}=\vec{Q} \cdot \bar{v}$.

$$
\mathrm{S}(\mathrm{p}) \cdot \overline{\mathrm{v}}^{2}=\overrightarrow{\mathrm{Q}} \cdot \bar{v} \approx \mathrm{N}, \mathrm{p} \cdot \mathrm{k}_{\mathrm{c}} \cdot \overline{\mathrm{v}}^{2} \approx \mathrm{N}
$$

where $N$ - capacity of interaction.

If to mean electromechanical analogies believing that $v \rightarrow i$ (velocity 
$\overline{\mathrm{V}}$ corresponds to a current in an electric chain) $S(p) \cdot i^{2} \rightarrow$ corresponds to full electric capacity. In this case comparison of an impedance to resistance of a chain is justified. This resistance is considered as full complex resistance. It has some components.

\section{Some questions of mechanical chains theory.}

Structural schemes of mechanical oscillatory systems can be various, dependences on a choice of the generalized co-ordinates which are used for the description of a dynamic condition of system and a kind of external influences. In the theory of electric chains as parameters of a dynamic condition of system pressure and currents are usually used. Created mathematical models of electric schemes look like electric chains in which as the basic typical elements are used inductance, resistors and capacities. In the linear theory of chains typical elements reflect idealized properties. In construction of electric schemes others are used also more difficult elements. The greatest interest for the linear theory is represented by transformers, as formations in relation to separate inductances. Let's notice that in electric chains the transformer breaks integrity of an electric chain that communication between two windings is provided any more with conductors, and through an electromagnetic field. In this respect interest is represented by electromechanical analogies of communications between chain elements. In particular it concerns working out for mechanical oscillatory systems of analogues in the form of various transformers. The attention to lever communications and mechanisms in problems of dynamics of mechanical oscillatory systems $[2,9,13,17]$ became one of such directions of research.

In the theory of electric chains concepts about typical elements which are based on inversion of characteristics of inductance, capacity and resistors $(1 / L, 1 / \mathrm{c}$ and $1 / r$ where designations of parameters have the standard form are used widely enough.

$$
\begin{aligned}
& \text { As to parity use } S(p)=\frac{k_{c}}{p} \text {, then of } \\
& \bar{y} \cdot k_{c}=\bar{Q}, \bar{y} \cdot k_{c} \cdot \bar{v}=N=p\left(y^{2}\right) N
\end{aligned}
$$

If $k=k$, then $k y^{2}=2 \Pi$ will represent of doubled potential energy.
I. At transition to mechanical oscillatory systems as to analogues of electric chains, two systems of analogies are used usually. The data about such systems of analogies is resulted, for example, in $[4,5]$. In the first system to force - there corresponds pressure, and to speed of points - there corresponds a current. In the second system of electromechanical analogies pressure corresponds to speed, and force - corresponds to a current. In dynamics of mechanical oscillatory systems use of the mentioned analogies quite explainable, in connection with simplicity of transition to definition of such parameters of a condition as efforts and speeds in separate points of system, signaling, an estimation of capacity of interactions. As sources of external energy in the theory of electric chains pressure and current generators are used. In mechanical oscillatory systems external indignations are presented by power and kinematic influences. The first represent known harmonious forces which are put to mass-inertial elements of system. Kinematic indignations represent known movements of basic surfaces to which typical elements of mechanical oscillatory systems contact more often. Structural interpretations of power and kinematic indignations at use of structural approaches are developed is not detailed enough, that in dynamic calculations to consider internal resistance of sources of indignation and limitation of their capacity. The greatest interest is represented by linear interpretations of interaction of sources of external influences with oscillatory systems.

II. Structural methods of research of mechanical oscillatory systems in approximations to problems of vibrating 
protection develop in directions of compatibility of approaches in use as device of the theory of automatic control, and methods of the theory of chains.

If mechanical oscillatory systems are interpreted by concepts of the theory of automatic control structural analogues lean against system of typical elements with transfer functions of intensifying devices and links with transfer functions of differentiating and integrating links of the first and second order.
At representation of mechanical oscillatory systems by structures of the theory of chains, typical elements are used also as well as in structural schemes of systems of automatic control equivalent in the dynamic relation. On fig. 4a-d possibilities of consideration of dynamic properties of systems in the different generalized co-ordinates are shown. On fig. 4b the structural scheme of mechanical oscillatory system (on fig. 4a), with a target signal in the form of displacement (is shown at the entrance signal having the form of force $Q$ ).

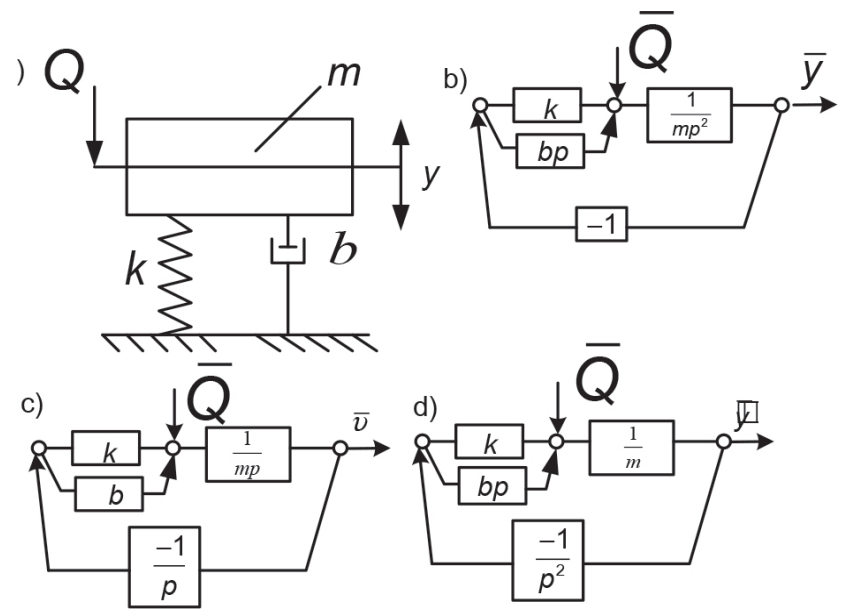

Fig. 4. Design (a) and structural schemes of mechanical oscillation system in different generalized coordinates: b) - coordinate of displacement $\overline{\mathrm{y}} ; c$ ) - coordinate of displacement $\overline{\ddot{\mathrm{y}}} ; d$ ) - coordinate of displacement $\ddot{\mathrm{y}}$;

If the target signal considers speed of object structural model (fig. 5c) will have other appearance. First of all it is connected by that the structure of typical elements changes. So, the object of protection $m$ will be presented by a link with transfer function of a typical integrating link of the first order. The elastic element will have transfer function $k(p)$ whereas on the structural scheme on fig. $4 b$, the elastic element has transfer function - $k$. In turn, dissipative the element on the structural scheme on fig. $4 \mathrm{c}$ has transfer function $\mathrm{b}$ whereas on fig. $4 \mathrm{~b}$ such link has transfer function $b p$ (on all schemes $\mathrm{b}$ corresponds to factor of a viscous friction).

At use as a target signal (fig. 4d) of acceleration $\overline{\ddot{y}}$, also will change structure of typical elements. In this case the object of protection will be presented by an intensifying link $1 / m$, the elastic element - will have transfer function - $k / p^{2}$, a dissipative link accordingly - $b / p$. It is important to notice that transformations of transfer functions of typical elements occurs according to transfer function of a chain of negative feedback. At an exit signal $\bar{y}$ feedback tie - individual (fig. 5 b). At an exit signal $\bar{v}=\overline{\dot{y}}$ in shape negative feedback is presented by an integrating link of the first sort (fig. 5c). As to a case at which the target signal represents acceleration $\overline{\ddot{y}}$ return negative communication has transfer function of an integrating link of the second order.

Let's consider variants of transfer functions at various target signals 


$$
\begin{aligned}
& W_{1}(p)=\frac{\bar{y}}{\bar{Q}}=\frac{1}{m p^{2}+b p+k}, \\
& W_{1}(p)=\frac{\bar{v}}{\bar{Q}}=\frac{\overline{\dot{y}}}{\bar{Q}}=\frac{1}{m p+b+\frac{k}{p}},
\end{aligned}
$$

\section{Using of different structural schemes in interpretations of representations as application to mechanical chains.}

For the design scheme on fig. 4a structural models with use of feedback ties are presented on fig. 4 b-d. Accordingly, on fig. 5 and, b, c

$$
W_{1}(p)=\frac{\overline{\ddot{y}}}{\bar{Q}}=\frac{1}{m+\frac{b}{p}+\frac{k}{p^{2}}} .
$$

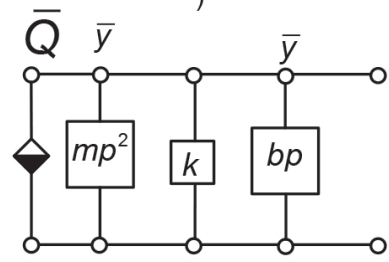

structural models of the mechanical chains, the having co-ordinate in a kind $\bar{y}, \bar{v}$ and $\bar{y}$ are resulted.

b)

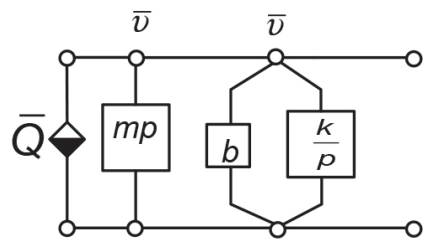

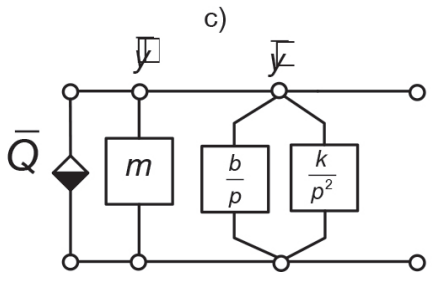

c)

Fig. 5. Variants of structural models of mechanical chain: a) - exit signal in view of coordinate $\overline{\mathbf{y}}$; b) - exit signal in view of coordinate $\overline{\mathrm{v}}$; c) - exit signal in view of coordinate $\overline{\ddot{y}}$

Thus transfer function (17) if to mean its physical sense, represents a pliability in a point of application of force $\bar{Q}$. It is reflected by an operational parity (17) which can be presented in a complex kind that can be received substitution $p=j \omega$. The pliability corresponds with displacement of a point of application of force that it is possible to interpret as the displacement applied on individual force.

If equation (17) to transform to a kind:

$$
\mathrm{k}_{\mathrm{c}}(\mathrm{p})=\frac{\overline{\mathrm{Q}}}{\overline{\mathrm{y}}}=\left(m \mathrm{p}^{2}+\mathrm{bp}+\mathrm{k}\right),
$$

that corresponds, as was already mentioned, the coerced stiffness of system on fig. 5a. In this case it is possible to present proceeding system as some generalized spring. Stiffness of this spring (20) depends on frequency of external influence. The coerced stiffness $k_{c}$ is dynamic rigidity. At constant force the coerced stiffness coincides with stiffness of a spring in 
a static condition $(k)$.

In physical sense the coerced stiffness at harmonious force $\bar{Q}$ reflects "resistance" of the system which condition is described by coordinate $\bar{y}$. In such situation force $\bar{Q}$ is put to object in weight $m$, the object gets the displacement having harmonious character. The maximum amplitude of fluctuations of object $(m)$ depends on system parameters, that is it mass-inertial and is elastic-dissipative properties. On each frequency the system will have the rigidity. In a general sense coerced stiffness depends on frequency.

By consideration of the same system (fig. 4a) which is described in the velocity $\bar{v}$ at force action $\bar{Q}$, the system condition is described by transfer function (18). In physical sense such transfer function reflects resistance of system on its movement with some speed. Such phenomenon usually name mobility. Having made inversion (18), we will receive kind equation:

$$
S(p)=\frac{\bar{Q}}{\bar{v}}=m p+b+\frac{k}{p} .
$$

This equation is named impedance. Physical sense of impedance is that he is identified of resistance of system when she gets certain velocity $\bar{v}$. Such situation will be other if speed of system isn't considered but his displacement $\bar{y}$. The last - is considered above and connected with definition of the coerced stiffness rigidity by equation (20). The impedance was named a full complex resistance in mechanical chains theory $[4,10,16]$. Definition of full complex resistance also is used in electrical chains theory $[4,5]$.

Development of approaches to theoretical and to experimental researches on the basis of impedances, has received enough wide circulation, in particular, under the relation to difficult systems.

If the system condition (fig. 4a) is estimated got at action of force $\bar{Q}$ by acceleration $\overline{\ddot{y}}$, it is estimated by transfer function (19).

The physical sense (19) consists that it characterizes system possibilities as a whole, to get in the established movement some acceleration caused by application of force $\bar{Q}$. Inversion of equation (19) allows the system characteristic to get acceleration that is estimated by the relation of force $\bar{Q}$ to acceleration in resistance rendering:

$$
R(p)=\frac{\bar{Q}}{\overline{\dot{y}}}=m+\frac{b}{p}+\frac{k}{p^{2}} .
$$

This property of system of response on force $\bar{Q}$ application can be named (as a proposal) harshness system. Acceleration of movement $\bar{y}$ on one of time is the harshness in physical sense. Parameter $R(p)$ is complex value. Comparison of equations (17)-(19) and (20)-(22) is shown that equation (21) impedance and connects with equation (20) and (22), in particular:

$$
\begin{aligned}
& k_{c}(p)=p S(p), \\
& R(p)=\frac{1}{p} S(p), \\
& R(p)=\frac{k_{c}(p)}{p^{2}}=\frac{S(p)}{p} .
\end{aligned}
$$

In turn, impedance $S(p)$ and harshness $R(p)$ can be identified over across coerced stiffness

$$
\begin{aligned}
& S(p)=\frac{k_{c}(p)}{p}, \\
& R(p)=\frac{k_{c}(p)}{p^{2}}=\frac{S(p)}{p} .
\end{aligned}
$$

Equations $(23) \div(27)$ are shown that impedance $S(\omega)$ isn't exclusive characteristic of oscillation system and connects with elastical definition (coerced stiffness, harshness). In turn impedance $S(\omega)$ is response characteristic of system on velocity of movement of application point. It can named velocity impedance. Then coerced stiffness complies displacement impedance and acceleration complies of speed-up impedance. Note that in [4] value $\wedge(p)=\frac{1}{S(p)}$ was named admittance.

Expedient of inversion of transfer 
functions for identification of coerced stiffness, impedance, harshness is used in previous charter. Some interest represents using of inversion.

\section{CONCLUSIONS.}

Definition of impedance connects with estimation technologies of dynamical properties of mechanical systems in which selected point of entrance action application in view of force with appropriate measurement of velocity of movement of mass-inertial element. Availability of structural interpretations of mathematical models of initial systems in view of structural schemes with adequacy reflection of physical properties of mechanical system makes impedance one of variants or form of transfer functions. Obtaining of transfer functions predetermines possibilities oflocation not only impedance but such parameters as movement acceleration or displacement itself. New characteristics connect with impedance of simple analytical ratios that let to transform of obtained information with distribution on other parameters of dynamical condition.
Structural schemes-analogs of mathematical models of mechanical systems reflect properties of feedback tie in setting of dynamical conditions of oscillation system and can be displayed of mechanical chains and be in equivalent forms in view of structures with assuming of mutual transitions with obtaining the same functional relations.

Each form of structural mathematical models has his features and potentially has possibilities of display of such properties in mechanical systems as energy transfer, interactions with energy sources, energy loss in the transmission of motion and etc.

If impedance approach bases on accounting of cardinality factors in dynamical interactions that application of displacements and accelerations will suggests using other forms of energy transformation.

\section{REFERENCES}

1. Babakov, I.M. Oscillations theory. - M.: Science, 1968. 650 p.

2. Belokobil'skiy, S.V., Eliseev, S.V., Kashuba, V.B. Applied tasks of the structural theory of vibroprotective systems. -SPb.; Politekhnika, 2013. - 364 p.

3. Belokobyl'sliy, S.V., Eliseev, S.V., Sitov, I.S. Dynamics of mechanical systems. Lever and inertial and elastic ties. - SPb.; Politekhnika, 2013. - 319 p.

4. Drujinskii, I.A. Mechanical chains. - M.: Mechanical engineering, 1977. - 238 p.

5. Gardner, F., Barns, J.L. Transient processes in linear systems with concentrated constants. - Moscow-Leningrad: GITTL, 1949. - 528 p.

6. Genkin, M.D., Ryaboi, V.M. Elastic-inertial vibroisolation systems. Limiting possibilities, optimal structures. - M.: Science,1988. - 191p.

7. Harris', C.M., Allan, G. Shock and Vibration Handbook. USA/Mc Graw-Hill, NewYork. 2002. - pp. 877.

8. Eliseev, S.V., Belokobil'skiy, S.V., Upir, R.Yu., Gozbenko, V.E. Lever ties in tasks of dynamics of mechanical oscillation systems. Theoretical aspects; IrGUPS. - Irkutsk, 2009, 158 p. Deposited in VINITI 27.11.2009 №737 - In 2009.

9. Eliseev S.V., Moskovskih A.O.,. Bolshakov R.S, Savchenko A.A. Possibilities of integration of methods of chains theory and automation control theory in tasks of machines dynamics // technomag.edu.ru: Science and education: electronic sciencetechnic edition. №6. 2012. URL. http:// technomag.edu.ru/doc/378699.html (date of appeal: 10.06.2012).

10. Eliseev, S.V., Reznik, Yu.N., Khomenko, A.P. Mechatronical approaches in dynamics 
of the mechanical oscillatory systems. - Novosibirsk: Nauka, 2011. - 394 p.

11. Eliseev, S.V., Reznik, Yu. N., Khomenko, A.P., Zasyadko, A.A. Dynamic synthesis in the generalized problems of vibroprotection and a vibration insulation of technical objects. - Irkutsk: publ. ISU, 2008. - 523 p.

12. Eliseev, S.V., Upir, R.Yu., G,ozbenko V.E. Modern situation of developments in the region of transport dynamics; IrGUPS. - Irkutsk, 2009, 128 p. Deposited in VINITI 27.11.2009 №739 - In 2009.

13. Khomenko, A.P., Eliseev, S.V. Dynamical absorbtion of oscillations: concept of feedback tie and structural methods of mathematical modeling. - Novosibirsk: Science, 2014. - 357 .

14. Khomenko, A.P., Eliseev, S.V. Quasi-elements in mechanical oscillation systems. Features of systems at exclusion of variables of dynamical condition / Modern technologies. System analysis. Modelling. 2013. № 2 (38). p. 8-17.

15. Khomenko, A.P., Eliseev, S.V., Artyunin, A.I., Parshuta, E.A., Kaimov, E.V. Mechanisms in elastic oscillatory systems: features of the accounting of dynamic properties, problems of vibration protection of machines, devices and equipment. IrGUPS. - Irkutsk, 2013, 187 p. Deposited in VINITI 15.08.2013 №243 - In 2013.

16. Kolovskii, M.Z. Automation control of vibroprotections systems / M.Z. Kolovskii. M.: Science, 1976. -320 p.

17. Panovko, G.Ya. Lectures on bases of theory of vibration machines and technologies: Tutorial for inversities. - M: Pub. house Bauman MSTU, 2008. - 192 p.

18. Vibrations in technics: handbook in 6 volumes. / Ed. board: V.N. Chelomey (pred.). - M.: Mechanical enguneering,1981. - V.5. Measuring and testing. - Ed. M.D. Genkin. 1981. -496 p. 